\title{
Material multimedia en español, valenciano e inglés para la clase práctica de Auscultación Cardíaca Multimedia material in Spanish, English and Valencian for the practical class of Cardiac Auscultation
}

\author{
María Pascual Mora ${ }^{1}$, Begoña Pineda Merlo ${ }^{1}$, Soraya Vallés Marti ${ }^{1}$, Adrián Jordá Vallés ${ }^{2}$, Martín Aldasoro Celaya ${ }^{1}$ \\ maria.pascual@uv.es, begona.pineda@uv.es, lilian.valles@uv.es, adrian.jorda@uv.es,martin.aldasoro@uv.es \\ ${ }^{1}$ Departamento de Fisiología \\ Facultad de Medicina y Odontología \\ Universidad de Valencia \\ Valencia, España \\ ${ }^{2}$ Departamento de Enfermería \\ Facultad de Enfermería y Podología \\ Universidad de Valencia \\ Valencia, España
}

\begin{abstract}
Resumen- En los últimos años, el uso de material multimedia se ha convertido en una práctica habitual en las aulas, ya que permite que los estudiantes mantengan y mejoren la atención en clase, así como una mayor comprensión de los conceptos adquiridos. Es por ello, que hemos llevado a cabo un vídeo en español, valenciano e inglés de la clase práctica de Auscultación Cardíaca en el Grado de Medicina y otros grados afines dentro de las Ciencias de la Salud. El vídeo se realizó en los tres idiomas utilizados (español, inglés y valenciano) en los grupos del Grado de Medicina. Se trata un procedimiento clínico de exploración física ampliamente utilizado en clínica, pero que al mismo tiempo presenta cierta dificultad para el estudiante. Los objetivos son aprender a localizar exactamente los focos de auscultación cardíaca (mitral, aórtico, tricuspídeo y pulmonar) y el reconocimiento de cada uno de los ruidos cardíacos. Este material multimedia permite que los estudiantes mantengan y mejoren la atención en clase y aumente su capacidad de aprendizaje, tal y como ha sido mostrado en las encuestas realizadas. Su uso en la actualidad supone una ayuda para los estudiantes que tengan que realizar esta práctica de modo no presencial.
\end{abstract}

Palabras clave: metodología docente, clase práctica, auscultación cardíaca, encuesta, vídeo.

Abstract- Recently, the use of multimedia material has become a common practice in the classes, as it allows that the students are able to maintain and improve class attention, as well as to understanding better all the concepts acquired. In this sense, we have carried out a video in Spanish, Valencian and English of the practical class of Cardiac Auscultation taught in the Medical Degree and in other related degrees within the Health Sciences. The video was made in the three languages used (Spanish, English and Valencian) in the Medicine Degree groups. It is a clinical physical examination procedure widely used in the clinic field, but at the same time it presents some difficulty for the student. The objectives are to learn to accurately locate the cardiac auscultation foci (mitral, aortic, tricuspid and pulmonary) and to recognize each of the heart sounds. This multimedia material allows students to maintain and improve attention in class and increase their learning capacity, as has been shown in the surveys carried out. Nowadays, its use is an aid for students who have to carry out this practice in a non-face-to-face way.
Keywords: teaching methodology, practical class, cardiac auscultation, survey, video.

\section{INTRODUCCIÓN}

La auscultación cardíaca es un procedimiento clínico de exploración física ampliamente utilizado en clínica, con el que se es capaz de diagnosticar gran parte de las patologías cardiovasculares congénitas, siendo comúnmente utilizado en el paciente pediátrico (Zipes \& Libby, 2018). Debido a ello, el conocimiento de esta técnica es imprescindible y de gran importancia para el estudiante del Grado de Medicina. La clase práctica de Auscultación Cardíaca pertenece al módulo de Cardiovascular de la asignatura de Fisiología Médica I del Grado de Medicina, y a otros grados afines en el área de las Ciencias de la Salud. Esta práctica presenta cierta dificultad para el estudiante debido a la localización exacta de los focos de auscultación y al reconocimiento de cada uno de los ruidos cardíacos. La utilización de un método de innovación docente (EDUTEC, 2009; López Zamora \& López Domínguez, 2013) basado en la proyección de un vídeo en el laboratorio en el que se muestre el modo de localizar los focos de auscultación valvular, es decir las zonas de expresión preferente para cada ruido cardíaco, y el reconocimiento de cada uno de los ruidos cardíacos fisiológicos (Zipes \& Libby, 2018), hará que el estudiante mejore el aprendizaje de dicha práctica, potenciando su atención y motivación en clase. Es por ello, que hemos llevado a cabo un vídeo en español, valenciano e inglés de la clase práctica de Auscultación Cardíaca, mediante la concesión de dos Proyectos de Innovación Docente concedidos por la Universitat de València. De este modo, la elaboración del vídeo en los tres idiomas facilita su proyección en cada uno de los grupos de español, valenciano e inglés del Grado de Medicina.

Dicho material multimedia está a disposición de los estudiantes en la plataforma de la Universitat de València, denominada Aula Virtual. Dicho material puede ser consultado por los estudiantes con antelación a la realización de la práctica, para que de esto modo puedan visualizarla antes de realizar la práctica, con el fin de que tengan una mayor comprensión de la explicación durante la práctica, un mayor aprendizaje $\mathrm{y}$ 
adquisición de los contenidos o incluso la posibilidad de la resolución de dudas surgidas durante la visualización previa del vídeo (EDUTEC, 2009; López Zamora \& López Domínguez, 2013). Por tanto, el alumno se familiariza con el material, la técnica y los conceptos que se van a utilizar durante la realización de la práctica en el laboratorio (Bates, 2017; Rozman, 1986). Asimismo, este material multimedia es utilizado en la explicación de la práctica, aportando una ayuda en el proceso didáctico (EDUTEC, 2009; López Zamora \& López Domínguez, 2013). Durante este curso académico 2020/21, este vídeo ha facilitado que la práctica pueda ser realizada de modo asíncrono o incluso de modo síncrono por los estudiantes que en ese momento no se encontraban en el aula.

Se puede considerar que la introducción en el sistema educativo de material multimedia, así como de otras Tecnologías de la Información y la Comunicación (TIC), supone un recurso válido para la mejora de la calidad docente, y nos ofrecen posibilidades y ventajas a tener en cuenta (López Zamora \& López Domínguez, 2013; Mirete, García-Sánchez, \& Hernández-Pina, 2015). Este video nos puede aportar flexibilidad y mejora en los procesos de enseñanza y aprendizaje (Mirete et al., 2015). Pero para seguir ahondando en las ventajas y mejoras que puede suponer el uso de este vídeo, se llevó a cabo su valoración mediante la realización de una encuesta para poder llevar a cabo una mejora en la calidad docente (Mirete et al., 2015).

\section{CONTEXTO}

El presente material multimedia es de gran ayuda para los estudiantes en el aprendizaje de la metodología de la Auscultación Cardíaca, puesto presenta cierta dificultad. Dicha dificultad se debe a que se han de localizar la posición exacta de los focos de auscultación cardíaca y el reconocimiento de cada uno de los ruidos cardíacos. Además, al ser una metodología muy habitual en clínica, de ahí que resulte sumamente importante un correcto aprendizaje de la técnica de auscultación cardíaca.

Los principales objetivos del vídeo son los siguientes:

1) Desde un punto de vista clínico, los estudiantes aprenden las partes de las que consta el estetoscopio biauricular (fonendoscopio) y el manejo adecuado del mismo. Además, aprenden a localizar los focos de auscultación valvular, es decir las zonas de expresión preferente para cada ruido cardíaco, así como el reconocimiento de cada uno de los ruidos cardíacos fisiológicos.

2) Desde un punto de vista docente, permite optimizar el tiempo en clase, ya que proporciona una mayor comprensión de los conceptos que son explicados en clase, dando lugar a menos dudas entre el alumnado sobre los objetivos propuestos y sobre su ejecución. Por tanto, puede repercutir positivamente en el tiempo establecido para la realización y desarrollo de la práctica.

3) En cuanto al aprendizaje por el alumnado, facilita que el vídeo pueda ser utilizado en cualquier momento, siendo utilizado para la preparación de los exámenes de la asignatura. Los conceptos al ser explicados de una forma más didáctica, mediante dibujos, animaciones o sonidos, pueden facilitar el aprendizaje de los conceptos impartidos en el vídeo. Todo ello favorece la adquisición de los conceptos, siendo éstos muy necesarios en los estudiantes de Medicina por la gran aplicabilidad de dicha técnica en la práctica clínica.

El vídeo puede ser utilizado por los estudiantes, no solo del grado de Medicina, sino de otros grados afines a las Ciencias de la Salud, como Farmacia, Fisioterapia, Enfermería y Ciencias de la Actividad Física y del Deporte.

\section{DESCRIPCIÓN}

El vídeo mejora la actividad docente en los grupos de español, inglés y valenciano, ya que queda reflejado el material y la metodología necesaria para llevar a cabo la práctica de "Auscultación cardíaca". Por tanto, el vídeo recoge los siguientes aspectos clínicos de la práctica:

1.- Introducción del fundamento de la práctica de "Auscultación cardíaca" y la importancia de su utilización en clínica (Bates, 2017).

El vídeo se inicia con la descripción de la ubicación de cada una de las zonas de expresión preferente para cada ruido o focos de auscultación valvular. Clásicamente han venido considerándose cuatro focos de auscultación cardíaca. Dado que los ruidos cardíacos primero y segundo, que son los normalmente audibles con el fonendoscopio, tienen componentes valvulares evidentes e importantes, se buscan aquellos puntos o focos de la pared anterior torácica, que aunque no representan los focos o zonas de proyección anatómica exacta de las válvulas cardíacas, se tratan de zonas donde se perciben mejor los ruidos cardíacos, lo cual no quiere decir que en cada uno de los focos de auscultación se vaya a oír solamente el ruido ocasionado por una sola válvula, sino que el ruido valvular que más intensamente se oiga es el que ocasiona la válvula en cuyo foco se esté auscultando. Esta técnica es de vital importancia en clínica ya que forma parte de la exploración física del paciente, con la que se es capaz de diagnosticar gran parte de las patologías cardiovasculares congénitas, siendo comúnmente utilizada en el paciente pediátrico (Bates, 2017; Zipes \& Libby, 2018).

\section{2.- Descripción de los cinco focos de auscultación.}

Los focos de auscultación no son las zonas exactas donde se proyectan las diferentes válvulas, es decir es donde mejor se oyen los diferentes ruidos (Zipes \& Libby, 2018).

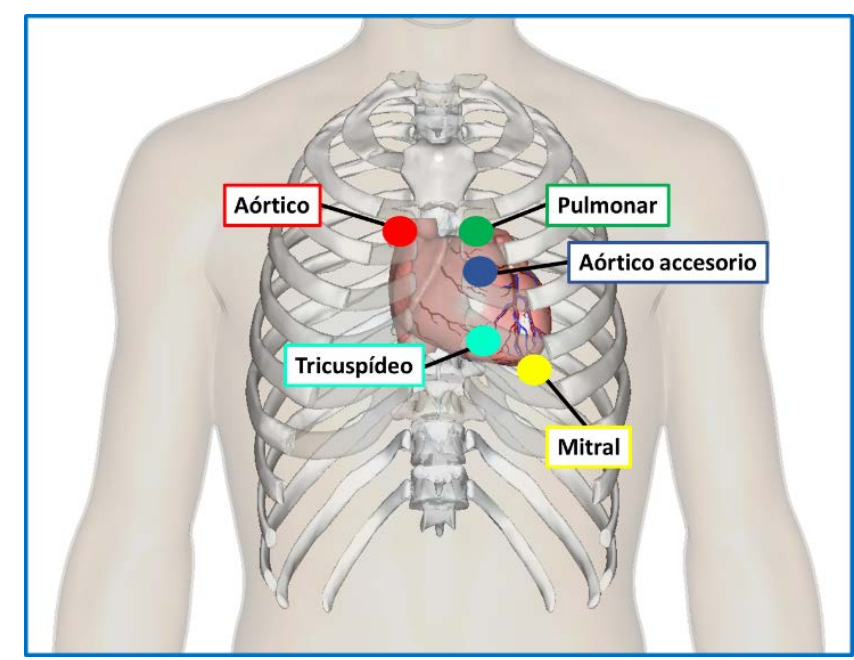

Figura 1. Localización de los focos de auscultación cardíaca. 
La Figura 1 muestra los cinco focos de auscultación cardíaca con su correcta localización en el tórax. Estos focos son los siguientes: foco mitral, foco aórtico, foco tricuspídeo, foco pulmonar, por ultimo también existe también el llamado "foco aórtico accesorio o secundario" (zona de Erb).

3.- Para realizar el presente material multimedia se necesita de una habitación a temperatura agradable, donde se "escuche el silencio", es decir sin ruidos, una cama clínica de reconocimiento y un estetoscopio biauricular o fonendoscopio (Rozman, 1986). En este caso, se ha de utilizar un fonendoscopio electrónico, en lugar de uno convencional, con la finalidad de poder grabar los ruidos cardíacos y su registro mediante una aplicación móvil, con el fin de facilitar la edición del vídeo (Fig. 2). En la grabación del vídeo, así como en su posterior edición se ha llevado a cabo por el personal técnico de innovación educativa (Servei de Formació Permanent i Innovació Educativa) de la Universidad de Valencia.

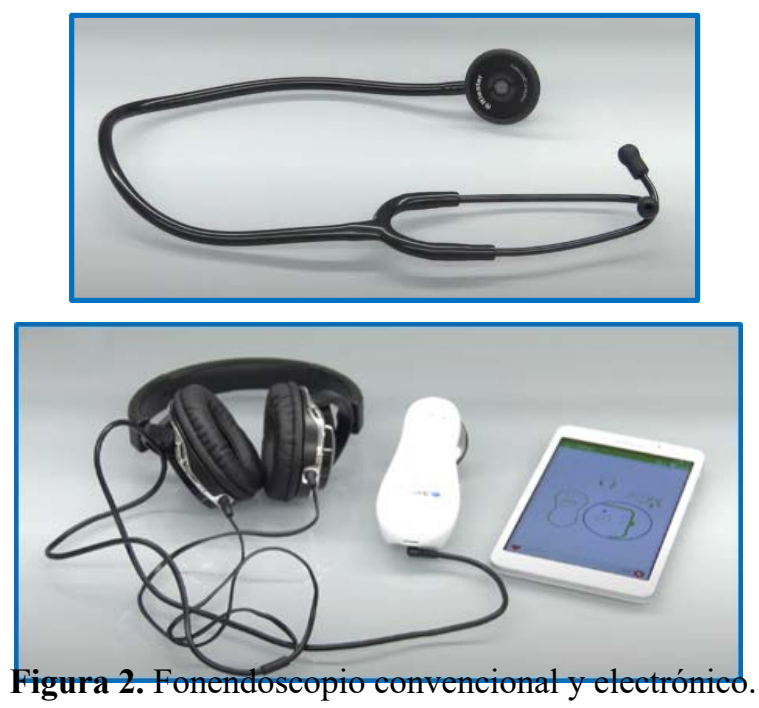

4.- Tras realizar una breve explicación de la posición que debe de adoptar el sujeto al que se le va a realizar la práctica de Auscultación Cardíaca (decúbito supino con la cabeza ligeramente incorporada), se procede a explicar los ruidos cardíacos (Zipes \& Libby, 2018). En la explicación de los cuatro ruidos cardíacos en el material multimedia se ha utilizado un montaje realizado con el programa Microsoft PowerPoint en el que se ilustra a través de diferentes diapositivas los aspectos físicos que dan lugar a cada uno de los tonos cardíacos.

5.- A continuación, se efectúa la auscultación en cada uno de los focos cardíacos, describiéndose en el vídeo de forma detallada cómo proceder con la auscultación de los focos cardíacos. Se lleva a cabo la auscultación cardíaca sobre un sujeto, encontrándose este lo más tranquilo y relajado posible. Como muestra la Figura 3, en cada uno de los focos de auscultación se detalla en la imagen del vídeo la localización exacta del foco a auscultar, mediante la superposición sobre el sujeto de un tórax y unos ejes de coordenadas, lo que permitirá que el estudiante sepa localizar el foco con más exactitud.

El procedimiento de la auscultación cardíaca es: 1) Auscultar con el diafragma primero y con la campana después o viceversa, en un determinado foco, centrando la atención en la percepción de un ruido primero, después en el otro y para finalizar se centra la atención en los dos ruidos eonjumtamente (Fig. 3). 2) Se repite el procedimiento en los demás focos de auscultación. 3) Para finalizar es interesante considerar determinadas variaciones con las que proceder de nuevo con la auscultación cardíaca como, por ejemplo: auscultación tras que el sujeto ha realizado una inspiración máxima seguida de apnea (parada ventilatoria), una espiración seguida de apnea o un ejercicio físico determinado.

6.- Por último, el material multimedia se evalúa mediante la realización de una encuesta anónima (Mirete et al., 2015),

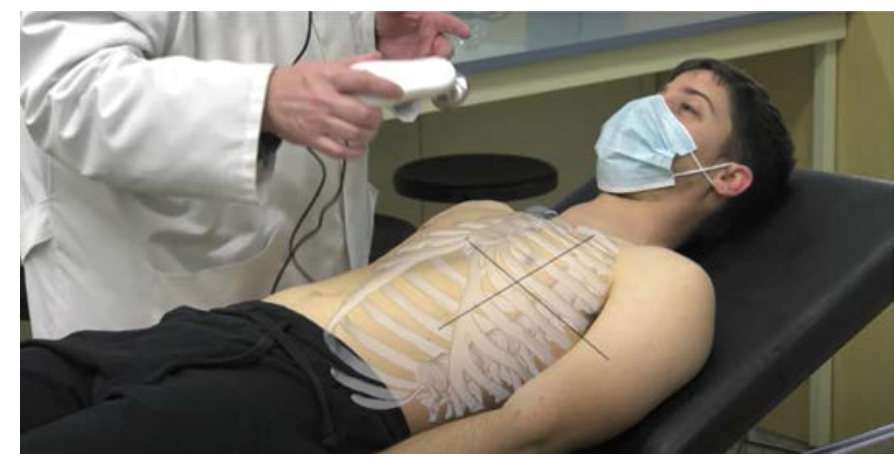

Figura 3. Imagen del vídeo en el que se observa en el paciente, la posición de uno de los focos de auscultación que a ser auscultado por el médico.

llevada a cabo a través de la plataforma de la Universidad de Valencia denominada "Aula Virtual". Esta encuesta es realizada a los estudiantes de los grupos de español, inglés y valenciano de la asignatura de Fisiología Médica I del Grado de Medicina. Se realizan una serie de cuestiones para poder analizar la utilidad del vídeo en el proceso de aprendizaje de los alumnos. Dicha encuesta consta de 4 preguntas: ¿te ha sido de utilidad para el aprendizaje de la práctica?, ¿te ha facilitado la comprensión del procedimiento explicado para la determinación de la auscultación cardíaca?, ¿crees que el vídeo te será de utilidad para la preparación del examen?, ¿recomendarías el video a otros compañeros/as, estudiantes de ciencias del área de la salud? Al mismo tiempo, al final de las cuestiones hay un pequeño espacio para que los estudiantes puedan dejar sus comentarios o sugerencias respecto al vídeo, para tratar de mejor el aspecto docente del vídeo en futuros cursos académicos.

\section{Resultados}

\section{1.- Elaboración del material multimedia}

La elaboración del material docente multimedia en español, inglés y valenciano para su incorporación en las prácticas de los temas de Cardiovascular de la asignatura de Fisiología Medica I del Grado de Medicina, con el fin de conseguir una mejora en la calidad docente en cada uno de los grupos de primer curso del grado, utilizándose en el vídeo la misma lengua que la utilizada durante la práctica. Del mismo modo, el material docente multimedia se puede difundir y aplicar a la asignatura de Fisiología en diferentes Grados de Ciencias de la Salud, como Farmacia, Fisioterapia, Nutrición Humana y Dietética, Podología, Enfermería y Ciencias de la Actividad Física y el Deporte.

El material docente elaborado en los 3 idiomas se encuentra en los enlaces "mmedia" y "YouTube" como se muestra en la Figura 4.

\section{Vídeo español:}

20-22 Octubre 2021, Madrid, ESPAÑA

VI Congreso Internacional sobre Aprendizaje, Innovación y Cooperación (CINAIC 2021) 
https://mmedia.uv.es/html5/g/cream/70186_auscultacioncardiac a16.mp4

https://youtu.be/5TbIdAY9CBE

\section{Vídeo inglés:}

https://mmedia.uv.es/html5/g/cream/70185_auscultacioncardiac aingles 16.mp4

https://youtu.be/30iIC_8E5QA

\section{Vídeo valenciano:}

https://mmedia.uv.es/html5/g/cream/70187_auscultacioncardiac avalencia16.mp4

https://youtu.be/F4inVzp1GYc

Figura 4. Imagen de los enlaces "mmedia" y "YouTube" de los 3 vídeos.

\section{2.- Análisis de las visualizaciones realizadas en "YouTube"}

Durante un periodo de 60 días se analizaron las descargas en los tres vídeos. Mientras el vídeo en español mostraba 1119 visualizaciones, 21 "me gusta" y 1 "no me gusta", en cambio el vídeo en inglés presentaba 4910 visualizaciones, 67 "me gusta" y 3 "no me gusta". Por otro lado, el vídeo en valenciano manifestaba 232 visualizaciones y 2 "me gusta".

Estos resultados nos sugieren que el vídeo más visto es el de inglés puesto que puede ser visualizado por estudiantes de otras universidades a nivel internacional. Por tanto, presenta un mayor número de "me gusta". Además, las visualizaciones presentadas en el vídeo en valenciano son mayor que el número de estudiantes que suele haber en dicho grupo (70-80 estudiantes), lo que sugieren que los estudiantes pudieron visualizarlo también en el periodo de preparación del examen de la asignatura.

\section{3.- Análisis de la encuesta anónima}

La Figura 5 muestra los resultados obtenidos de la encuesta del vídeo realizada de forma anónima a los estudiantes de los grupos de español, inglés y valenciano. En cuanto a la primera pregunta se puede observar que cerca del $63-75 \%$ de los estudiantes que han visto el vídeo en los 3 grupos les ha sido de utilidad para el aprendizaje de la práctica. Entre un 17-25\% no saben si les ha sido de utilidad el vídeo y un $11 \%$ del grupo de inglés no ha visto el vídeo, y será este un porcentaje que se mantenga también en el resto de preguntas.

En la segunda cuestión planteada en la encuesta, a la gran mayoría de los estudiantes encuestados que han visto el vídeo les ha facilitado la explicación de la práctica.

En relación a la tercera pregunta planteada, entre el 63-75\% de los estudiantes piensa que les será de utilidad el vídeo en la preparación del examen. En cambio, entre $17-25 \%$ de los encuestados piensan que no lo sabe y sorprende que entre un 6 y $12 \%$ en los grupos de inglés y valenciano piense que no les será de utilidad para el examen, cuando en el vídeo se describen y detallan mediante explicación teórica y gráfica conceptos que han sido previamente impartidos en las clases teóricas.

Por último, en la gran mayoría de los estudiantes encuestados de los grupos de español y valenciano recomendarían el vídeo a otros estudiantes. Por otro lado, en el grupo de inglés, el 23\% no sabe si recomendaría el vídeo y $9 \%$ no lo ha visto.
4.- Análisis de los comentarios o sugerencias realizadas por $\underline{\text { los estudiantes en la encuesta }}$

En cuanto a los comentarios o sugerencias realizadas por los estudiantes de forma anónima acerca del vídeo, se puede observar que la gran mayoría de estudiantes expresa que les ha gustado el vídeo. Del mismo modo, también manifiestan que es muy didáctico y les ha servido como método de aprendizaje.

Algunos de los estudiantes manifiestan que ha sido uno de los vídeos que más les ha gustado y más utilidad ha tenido en su aprendizaje de la asignatura de Fisiología Médica I del Grado de Medicina.

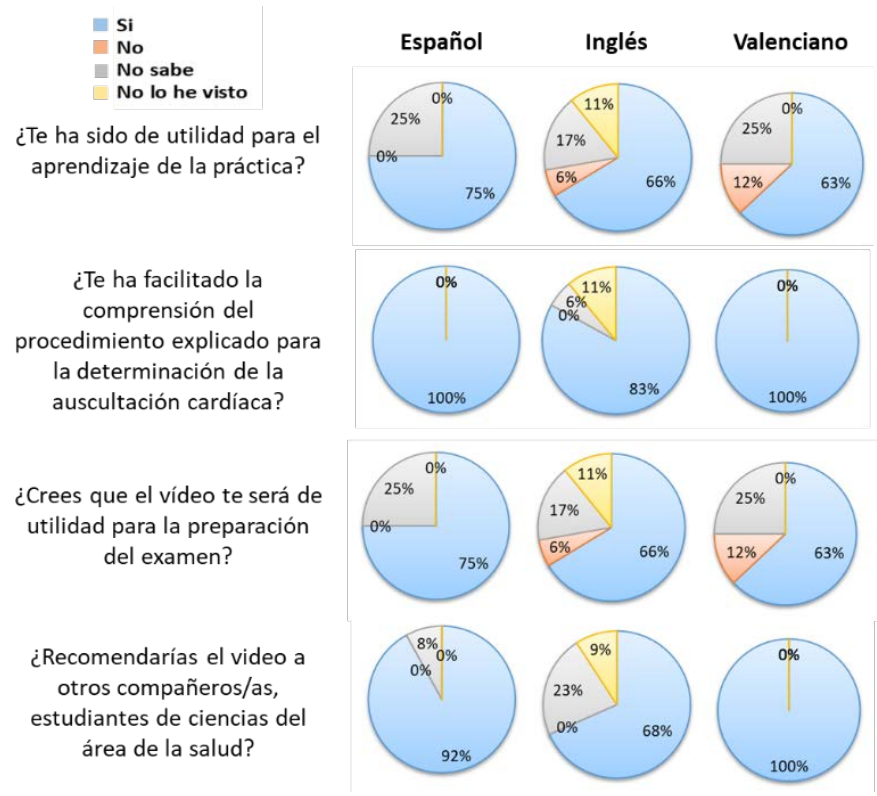

Figura 5. Resultados de la encuesta realizada a los estudiantes para valorar la utilidad del vídeo en el proceso de aprendizaje en la práctica de Auscultación Cardíaca.

\section{5.- Resultados a nivel académico del material multimedia}

Con la incorporación de este material multimedia en clase obtenemos en las clases prácticas un modelo docente más evolucionado, que mejora al modelo docente actual, mediante la incorporación de elementos complementarios, como el presente vídeo, proporcionando un aprendizaje más dinámico y promoviendo la atención, la motivación, la reflexión y las acciones del alumnado.

Al mismo tiempo, permite reforzar la comprensión de los conceptos de Fisiología referentes a la práctica en cuestión (localización de las válvulas cardíacas y las zonas de proyección de los ruidos o focos cardíacos). Por tanto, permitirá una mejor preparación tanto de cara al examen, como en un futuro en la práctica clínica de dicha técnica.

El vídeo favorece la optimización del tiempo en clase. Con el presente material multimedia se favorece que haya una mayor comprensión de los conceptos que son explicados en clase, dando lugar a menos dudas entre el alumnado sobre los objetivos propuestos y sobre su ejecución, y que todo ello repercute de forma positiva en el tiempo establecido para la realización de la práctica.

\section{CONCLUSIONES}


La utilización de esta metodología para la clase práctica de Auscultación Cardíaca en los grupos de español, inglés y valenciano ha tenido una gran acogida y les ha gustado mucho, como queda reflejado en las encuestas y comentarios o sugerencias realizadas por los estudiantes.

El vídeo ha sido utilizado por los estudiantes, no solo del grado de Medicina, sino de otros grados como Farmacia, Fisioterapia, Enfermería, Ciencias de la Actividad Física y del Deporte. De ello, se concluye su gran utilidad en la docencia de nuestro departamento.

Se ha garantizado que el vídeo permite optimizar mejor el tiempo en clase, ya que facilita una mayor comprensión de los conceptos que son explicados en clase.

De la docencia llevada a cabo en el presente curso académico 2020/2021, se ha confirmado que es una metodología muy útil tanto para la docencia presencial como para la no presencial. Por ejemplo, los estudiantes de Medicina pueden llevar a cabo la auscultación cardíaca con sus propios fonendoscopios en sus domicilios.

Ha sido de gran utilidad en la docencia práctica y teórica, puesto que es un procedimiento habitual en clínica y por ello ha de ser aprendido correctamente para llevarlo a cabo con gran precisión.

\section{AgRADECIMIENTOS}

El material multimedia de este trabajo se ha llevado a cabo gracias a concesión de proyectos de innovación docente de la Universidad de Valencia (UV-SFPIE_PID19-1097274 y UVSFPIE_PID20-1353113). Nos gustaría agradecer la ayuda del Prof. Francisco Javier Chorro Gascó (Jefe del Servicio de Cardiología del Hospital Clínico Universitario de Valencia y
Catedrático del Departamento de Medicina, Facultad de Medicina y Odontología, Universitat de València) y del Prof. Luis Such Belenguer (Prof. Emérito del Departamento de Fisiología, Facultad de Medicina y Odontología, Universitat de València) en la elaboración del vídeo. También nos gustaría agradecer al estudiante de Medicina, Fernando Roca Valderas, por su participación en el vídeo como paciente.

\section{REFERENCIAS}

Bates, B. (2017). Guía de exploración física e historia clínica. Barcelona: Wolters Kluwer-Lippincott.

EDUTEC. (2009). Ventajas del uso de las TICs en el proceso de enseñanza aprendizaje desde la óptica de los docentes universitarios españoles. 29 Julio.

López Zamora, I., \& López Domínguez, R. (2013). Las TICs como medio para el desarrollo de comunidades de aprendizaje en ciencias: el caso de los contenidos y la motivación. Revista iberoamericana para la investigación y el desarrollo educativo.

Mirete, A. B., García-Sánchez, F. A., \& Hernández-Pina, F. (2015). Cuestionario para el estudio de la actitud, el conocimiento y el uso de TIC (ACUTIC) en Educación Superior. Estudio de fiabilidad y validez. Revista interuniversitaria de formación del profesorado,, 83, 7589.

Rozman, C. (1986). Semiología y métodos de exploración en medicina. Barcelona: Salvat.

Zipes, D., \& Libby, P. (2018). Braunwald's Heart Disease: a textbook of cardiovascular medicine. Philadelphia: Saunders. 